\title{
THE EVOLUTION OF FAR-INFRARED CO EMISSION FROM PROTOSTARS
}

\author{
P. Manoj ${ }^{1}$, J. D. Green ${ }^{2}$, S. T. Megeath ${ }^{3}$, N. J. Evans II ${ }^{4}$, A. M. Stutz ${ }^{5}$, J. J. Tobin ${ }^{6}$, D. M. Watson ${ }^{7}$, W. J. Fischer ${ }^{8}$, \\ E. FURLAN ${ }^{9}$, AND T. HENNING ${ }^{5}$ \\ ${ }^{1}$ Tata Institute of Fundamental Research, Homi Bhabha Road, Mumbai 400 005, India; manoj.puravankara@tifr.res.in \\ ${ }^{2}$ Space Telescope Science Institute, Baltimore, MD, USA \\ ${ }^{3}$ Department of Physics and Astronomy, University of Toledo, 2801 West Bancroft Street, OH 43606, USA \\ ${ }^{4}$ The University of Texas at Austin, Department of Astronomy, 2515 Speedway, Stop C1400, Austin, TX 78712-1205, USA \\ ${ }^{5}$ Max-Planck-Institute for Astronomy, Königstuhl 17, D-69117 Heidelberg, Germany \\ ${ }^{6}$ Leiden Observatory, Leiden University, P.O. Box 9513, 2300-RA Leiden, The Netherlands \\ ${ }^{7}$ Department of Physics and Astronomy, University of Rochester, Rochester, NY 14627, USA \\ ${ }^{8}$ NASA Goddard Space Flight Center, Greenbelt, MD, USA \\ ${ }^{9}$ Infrared Processing and Analysis Center, California Institute of Technology, 770 S. Wilson Avenue, Pasadena, CA 91125, USA \\ Received 2016 May 16; revised 2016 August 11; accepted 2016 August 13; published 2016 October 27
}

\begin{abstract}
We investigate the evolution of far-IR CO emission from protostars observed with Herschel/PACS for 50 sources from the combined sample of HOPS and DIGIT Herschel key programs. From the uniformly sampled spectral energy distributions, whose peaks are well sampled, we computed the $L_{\mathrm{bol}}, T_{\mathrm{bol}}$, and $L_{\mathrm{bol}} / L_{\mathrm{smm}}$ for these sources to search for correlations between far-IR CO emission and protostellar properties. We find a strong and tight correlation between far-IR CO luminosity $\left(L_{\mathrm{CO}}^{\mathrm{fir}}\right)$ and the bolometric luminosity $\left(L_{\mathrm{bol}}\right)$ of the protostars with $L_{\mathrm{CO}}^{\text {fir }} \propto L_{\mathrm{boi}}^{0.7}$. We, however, do not find a strong correlation between $L_{\mathrm{CO}}^{\text {fir }}$ and protostellar evolutionary indicators, $T_{\mathrm{bol}}$ and $L_{\mathrm{bol}} / L_{\mathrm{smm}}$. FIR CO emission from protostars traces the currently shocked gas by jets/outflows, and farIR CO luminosity, $L_{\mathrm{CO}}^{\mathrm{fir}}$, is proportional to the instantaneous mass-loss rate, $\dot{M}_{\text {out }}$. The correlation between $L_{\mathrm{CO}}^{\mathrm{fir}}$ and $L_{\text {bol }}$, then, is indicative of instantaneous $\dot{M}_{\text {out }}$ tracking instantaneous $\dot{M}_{\text {acc }}$. The lack of a correlation between $L_{\mathrm{CO}}^{\text {fir }}$ and evolutionary indicators $T_{\text {bol }}$ and $L_{\text {bol }} / L_{\mathrm{smm}}$ suggests that $\dot{M}_{\text {out }}$ and, therefore, $\dot{M}_{\text {acc }}$ do not show any clear evolutionary trend. These results are consistent with mass accretion/ejection in protostars being episodic. Taken together with the previous finding that the time-averaged mass-ejection/accretion rate declines during the protostellar phase, our results suggest that the instantaneous accretion/ejection rate of protostars is highly time variable and episodic, but the amplitude and/or frequency of this variability decreases with time such that the timeaveraged accretion/ejection rate declines with system age.
\end{abstract}

Key words: ISM: jets and outflows - stars: jets - stars: protostars - stars: winds, outflows

\section{INTRODUCTION}

Mass accretion in young stellar objects is thought to be highly time variable and episodic (e.g., Kenyon et al. 1990; Hartmann \& Kenyon 1996; Evans et al. 2009; Hartmann 2009; Dunham \& Vorobyov 2012). Outbursts of varying intensities and frequencies have been observed in several young stars (e.g., Herbig 1977; Kenyon 1995; Green et al. 2006; Reipurth \& Aspin 2010; Fischer et al. 2012; Green et al. 2013a; Audard et al. 2014; Safron et al. 2015). However, a detailed picture of the time evolution of mass accretion from the early protostellar phase to the late pre-main-sequence phase is still missing. Most of the commonly used direct observational tracers of mass accretion fall at wavelengths $\lesssim 2 \mu \mathrm{m}$ (e.g., Calvet \& Gullbring 1998; Muzerolle et al. 1998a, 1998b, 2001). While these tracers are used extensively to study the accretion history in pre-main-sequence stars (e.g., Gullbring et al. 1998; Hartmann 1998; Calvet et al. 2004; Muzerolle et al. 2004; Herczeg \& Hillenbrand 2008), they are difficult to observe in protostars that are deeply embedded in their natal core as their shorter wavelength emission is heavily extinguished.

Mass accretion in protostars is thought to be associated with mass ejection. Jets and outflows from embedded protostars are more readily accessible to observations than the direct accretion tracers, particularly at far-IR and (sub)millimeter wavelengths. Observations of protostellar jets and outflows at these wavelengths provide important diagnostics for the energetics of mass ejection and mass-loss rates from protostars (e.g., Watson 1985; Hollenbach et al. 1989; Bachiller \& Tafalla 1999; Richer et al. 2000; Nisini et al. 2015; Tobin et al. 2016). Moreover, theoretical models of mass ejection mechanisms from protostars predict a linear relation between mass-loss rate from protostars, $\dot{M}_{\text {out }}$, and mass-accretion rate, $\dot{M}_{\text {acc }}$, onto the protostar (Pelletier \& Pudritz 1992; Wardle \& Koenigl 1993; Najita \& Shu 1994; Shu et al. 1994; Matt \& Pudritz 2005, 2008). Thus, observed properties of mass ejection can be used to study the mass-accretion history in protostars.

While jets and outflows from protostars have been studied using several different tracers and at various wavelengths (e.g., Bally et al. 2007; Frank et al. 2014), the observational tool that is most often used for the largest sample of protostars is the low- $J\left(J_{\text {up }} \leqslant 3\right)$ CO lines at (sub-)millimeter wavelengths, which trace the ambient molecular gas swept up and accelerated by the protostellar jets (e.g., Bachiller \& Tafalla 1999; Richer et al. 2000; Arce et al. 2007; Hatchell et al. 2007a; Takahashi et al. 2008; Curtis et al. 2010; Dunham et al. 2014a; Plunkett et al. 2015). These observations of molecular outflows from protostars have shown that the timeaveraged flow energetic parameters, viz., the mechanical luminosity $\left(L_{\text {mech }}\right)$ and the momentum flux or outflow force $\left(F_{\mathrm{CO}}\right)$, are correlated with the bolometric luminosity of the protostar, $L_{\text {bol }}$ (Rodriguez et al. 1982; Bally \& Lada 1983; Lada 1985; Snell 1987; Cabrit \& Bertout 1992; Bontemps et al. 1996; Wu et al. 2004; Hatchell et al. 2007a; Takahashi 
et al. 2008; Curtis et al. 2010). A few of these studies also found an evolutionary trend in the outflow force $\left(F_{\mathrm{CO}}\right)$ with protostellar age (Bontemps et al. 1996; Curtis et al. 2010). Younger Class 0 sources are found to have more powerful outflows than the more evolved Class I protostars and the outflow power is found to decrease with system age. This has been interpreted as being due to a corresponding steady decline in the mass-accretion rate with time during the protostellar phase (Bontemps et al. 1996).

Emission lines due to the rotational transitions of $\mathrm{CO}$ in the far-IR (14 $\leqslant J_{\text {up }} \leqslant 45$ ), observed with the PACS instrument on board the Herschel space telescope, provide an alternate diagnostic of the jets/outflow properties of protostars. Unlike the low- $J$ CO lines observed at (sub)millimeter wavelengths, which trace the ambient molecular gas swept up by the jets/ outflows, the far-IR CO lines trace the hot gas that is currently being shocked by the jets/outflows from protostars. While the low- $J$ CO lines provide time-averaged energetics of the jets, the far-IR lines provide the instantaneous energetics of the jets. In this paper, we investigate the evolution of jet/outflow properties derived from the far-IR CO line luminosities for a large sample of protostars observed with Herschel/PACS as part of the two Herschel key programs (KP), the Herschel Orion Protostar Survey (HOPS) and the Dust, Ice, and Gas in Time (DIGIT).

\section{THE SAMPLE}

We analyzed the combined HOPS (Manoj et al. 2013) and DIGIT (Green et al. 2013b) sample of protostars for which we have Herschel/PACS spectra to search for evolutionary trends in the far-IR CO emission observed toward protostars. The HOPS program was a far-IR survey of Spitzeridentified protostars in the Orion molecular clouds. The HOPS team obtained and analyzed Herschel/PACS photometry of 330 protostars and PACS spectra of 36 protostars (Fischer et al. 2010, 2013; Stanke et al. 2010; Manoj et al. 2013; Stutz et al. 2013; Furlan et al. 2016; Gonzalez-Garcia et al. 2016). A detailed analysis of the far-IR spectra of the 21 brightest sources in the HOPS spectroscopy sample were presented in Manoj et al. (2013). From the Herschel/PACS imaging data, the HOPS team has also identified and characterized 16 new protostars in Orion, which were not detected by Spitzer or were too faint at mid-IR wavelengths (Stutz et al. 2013; Tobin et al. 2015). These sources, which are the reddest, and potentially youngest protostars in the Orion molecular clouds were called PACS Bright Red sources (PBRs; Stutz et al. 2013). Herschel/ PACS spectra for eight PBRs were later obtained by the HOPS team as part of the Herschel open time (OT) program (Tobin et al. 2016). The DIGIT program surveyed 94 young stellar objects in different evolutionary stages with Herschel and obtained far-IR spectra of 24 Herbig Ae/Be stars, 40 T Tauri stars, and 30 protostars in nearby $(d \lesssim 400$ pc) star-forming regions (van Kempen et al. 2010; Cieza et al. 2013; Dionatos et al. 2013; Fedele et al. 2013; Green et al. 2013b, 2013a, 2016; Meeus et al. 2013; Sturm et al. 2013; Lee et al. 2014b, 2014a). The Herschel/PACS spectra of 30 protostars in the DIGIT sample were presented in Green et al. (2013b).

All of the 21 HOPS protostars whose far-IR spectra were presented in Manoj et al. (2013) are inlcuded in the current analysis. However, only 21 of the 30 DIGIT sources presented in Green et al. (2013b) are considered here. The sources in the DIGIT sample are, on average, much closer than the HOPS sources, which complicates the sample in two ways: the sources are slightly extended at various wavelengths (depending on the envelope temperature structure) and some of the source fields (e.g., RCrA, Serpens) are crowded. The improved data pipeline (see Green et al. 2016) including jitter correction can successfully correct for a slightly extended source continuum, but only for the brighter sources, and only when the target is the dominant source in the field of view, within a spaxel of the center. It also assumes that the line emission is distributed identically to the continuum. The remaining nine DIGIT sources did not meet these criteria and are excluded; this includes the complicated regions RCrA-IRS5A, 7B and 7C, and Serpens-SMM3/4, the chain of MM sources in the L1448 field, and the off-center IRS 46/44 field. Also included in our analysis are the eight PBRs for which Herschel/PACS farIR spectra have been obtained (Tobin et al. 2016). Our final sample consists of a total of 50 protostars, which are listed in Table 1.

\section{DATA AND ANALYSIS \\ 3.1. Spectral Energy Distributions}

We first constructed the observed spectral energy distributions (SEDs) of our sample sources in order to estimate the basic protostellar properties. The SEDs of all the sources in our sample are uniformly sampled, from near-IR to the submillimeter/millimeter. For all sources, we have used Herschel/ PACS 70, 100, and $160 \mu \mathrm{m}$ fluxes to make sure that the peak of the protostellar SED is well sampled. Figure 1 shows a few representative SEDs. Below, we provide a detailed description of the photometric data used in the construction of the SEDs of the sources in our sample.

\subsubsection{HOPS}

For the HOPS sample, the 1.2 to $870 \mu \mathrm{m}$ photometric data used are from 2MASS, Spitzer IRAC, MIPS and IRS, Herschel PACS, and the Atacama Pathfinder Experiment (APEX; Manoj et al. 2013; Stutz et al. 2013; Furlan et al. 2016). The Spitzer IRS spectra have been rebinned to 12 flux values before integrating the SEDs. The details of the source of the photometry and the apertures used to extract the flux are described in the Appendix of Manoj et al. (2013). However, there are a few differences in the photometric data used in this analysis and those presented in Manoj et al. (2013). The primary one is in the PACS photometry: for several sources in crowded regions and/or regions where significant extended emission is present, we have updated the 70 and $160 \mu \mathrm{m}$ flux using point-spread function (PSF) photometry; in addition, for sources that have been observed multiple times in overlapping fields, the averaged value of the flux density is quoted (Furlan et al. 2016, B. Ali et al. 2016, in preparation). The PACS $70 \mu \mathrm{m}$ fluxes used in this analysis differ from those presented in Manoj et al. (2013) by $\sim 4 \%$ (median); for $75 \%$ of the sources, the difference is within $11 \%$. However, for two sources, HOPS 108 and 369 , the $70 \mu \mathrm{m}$ flux is lower by more than $60 \%$. These sources are in the crowded OMC-2 region, which also has significant extended emission and we have used PSF photometry to update the aperture photometry reported in Manoj et al. (2013). The median change in the PACS $160 \mu \mathrm{m}$ flux is $\sim 9 \%$; for $75 \%$ of the sources, the difference is within $20 \%$. For 11 sources, we have updated the $160 \mu \mathrm{m}$ flux using PSF photometry. The median difference from those presented in 
Table 1

Protostellar Properties

\begin{tabular}{|c|c|c|c|c|c|}
\hline $\begin{array}{l}\text { Object } \\
\text { Name }\end{array}$ & $\begin{array}{l}L_{\text {bol }} \\
\left(L_{\odot}\right)\end{array}$ & $\begin{array}{l}T_{\mathrm{bol}} \\
(\mathrm{K})\end{array}$ & $L_{\mathrm{bol}} / L_{\mathrm{smm}}$ & $\begin{array}{c}L_{\mathrm{CO}}^{\text {fir }} \\
\left(\times 10^{-3} L_{\odot}\right)\end{array}$ & $\begin{array}{c}L_{\mathrm{CO}(\mathrm{J}=14-13)} \\
\left(\times 10^{-3} L_{\odot}\right)\end{array}$ \\
\hline \multicolumn{6}{|c|}{ HOPS } \\
\hline HOPS 10 & 3.3 & 46 & 44 & 1.3 & 0.24 \\
\hline HOPS 11 & 8.9 & 47 & 80 & $\leqslant 1.2$ & $\leqslant 0.17$ \\
\hline HOPS 30 & 3.7 & 77 & 91 & $\leqslant 1.5$ & $\leqslant 0.18$ \\
\hline HOPS 32 & 2.1 & 60 & 117 & 1.8 & 0.21 \\
\hline HOPS 56 & 22.9 & 45 & 104 & 8.2 & 1.31 \\
\hline HOPS 60 & 22.0 & 55 & 127 & 6.2 & 0.95 \\
\hline HOPS 68 & 5.3 & 88 & 27 & 1.7 & 0.59 \\
\hline HOPS 84 & 49.0 & 90 & 206 & 0.7 & 0.30 \\
\hline HOPS 85 & 16.1 & 166 & 171 & 1.2 & 0.29 \\
\hline HOPS 87 & 36.3 & 38 & 54 & 7.6 & 0.98 \\
\hline HOPS 91 & 4.2 & 37 & 25 & $\leqslant 1.3$ & $\leqslant 0.19$ \\
\hline HOPS 108 & 36.3 & 34 & 47 & 237.7 & 18.13 \\
\hline HOPS 182 & 70.2 & 51 & 157 & 48.4 & 5.08 \\
\hline HOPS 203 & 19.8 & 41 & 72 & 9.7 & 0.82 \\
\hline HOPS 288 & 135.6 & 49 & 303 & 10.0 & 1.44 \\
\hline HOPS 310 & 13.8 & 51 & 121 & 9.8 & 1.24 \\
\hline HOPS 329 & 2.7 & 79 & 94 & $\leqslant 1.3$ & $\leqslant 0.19$ \\
\hline HOPS 343 & 3.8 & 79 & 188 & 2.0 & 0.23 \\
\hline HOPS 368 & 63.9 & 150 & 691 & 6.4 & 1.03 \\
\hline HOPS 369 & 18.0 & 35 & 74 & 11.0 & 1.85 \\
\hline HOPS 370 & 275.0 & 74 & 697 & 58.0 & 5.24 \\
\hline \multicolumn{6}{|c|}{ DIGIT } \\
\hline IRAS $03245+3002$ & 6.6 & 48 & 75 & 1.3 & 0.20 \\
\hline L1455-IRS3 & 0.3 & 236 & 16 & 0.1 & 0.06 \\
\hline IRAS $03301+3111$ & 4.5 & 349 & 186 & 0.9 & 0.09 \\
\hline $\mathrm{B} 1-\mathrm{a}$ & 1.5 & 113 & 285 & 1.6 & 0.23 \\
\hline B1-c & 3.2 & 46 & 439 & 0.9 & 0.23 \\
\hline L1489 & 3.5 & 248 & 145 & 0.8 & 0.07 \\
\hline IRAM $04191+1522$ & 0.1 & 15 & 50 & 0.2 & 0.03 \\
\hline L1551-IRS5 & 22.9 & 108 & 149 & 1.6 & 0.21 \\
\hline L1527 & 1.6 & 79 & 32 & 0.3 & 0.04 \\
\hline TMR 1 & 4.0 & 151 & 191 & 1.0 & 0.11 \\
\hline TMC 1A & 2.6 & 189 & 120 & 0.2 & 0.06 \\
\hline TMC 1 & 0.7 & 161 & 33 & 0.6 & 0.05 \\
\hline BHR 71 & 11.4 & 45 & 40 & 9.3 & 0.78 \\
\hline DK Cha & 28.3 & 605 & 655 & 4.4 & 0.41 \\
\hline GSS30-IRS1 & 10.6 & 172 & 432 & 5.7 & 0.65 \\
\hline VLA $1623-243$ & 3.3 & 27 & 23 & 2.0 & 0.31 \\
\hline WL12 & 1.6 & 236 & 57 & 1.1 & 0.10 \\
\hline Elias 29 & 15.2 & 310 & 528 & 6.3 & 0.60 \\
\hline B335 & 0.8 & 33 & 20 & 0.6 & 0.08 \\
\hline L1157 & 6.7 & 35 & 30 & 6.1 & 0.71 \\
\hline L1014 & 0.3 & 47 & 9 & $\leqslant 0.1$ & $\leqslant 0.02$ \\
\hline \multicolumn{6}{|c|}{ PBRs } \\
\hline HOPS 373 & 5.3 & 37 & 34 & 21.8 & 1.88 \\
\hline HOPS 394 & 6.5 & 45 & 38 & 1.9 & 0.31 \\
\hline HOPS 397 & 1.7 & 45 & 40 & 0.2 & 0.22 \\
\hline HOPS 401 & 0.6 & 26 & 13 & $\leqslant 1.0$ & $\leqslant 0.10$ \\
\hline HOPS 402 & 0.6 & 24 & 9 & $\leqslant 1.1$ & $\leqslant 0.09$ \\
\hline HOPS 403 & 4.1 & 44 & 34 & 2.5 & 0.35 \\
\hline HOPS 405 & 1.6 & 35 & 28 & 1.4 & 0.23 \\
\hline HOPS 409 & 8.3 & 28 & 47 & 5.8 & 0.76 \\
\hline
\end{tabular}

Manoj et al. (2013) is $11 \%$ and the maximum differences are in the cases of HOPS 91 and 108, which have their $160 \mu \mathrm{m}$ flux lower by $\sim 45 \%$ compared to that in Manoj et al. (2013). In this analysis, we have also added PACS $100 \mu \mathrm{m}$ photometry for our sources obtained from the Gould Belt Survey (e.g., André et al. 2010). The aperture used to extract the the $100 \mu \mathrm{m}$ flux is the same as that used for $70 \mu \mathrm{m}$ (for details, see Furlan et al. 2016). The 350 and $870 \mu \mathrm{m}$ data from APEX SABOCA and LABOCA were also re-reduced and re-calibrated (Stutz et al. 2013; Stutz \& Kainulainen 2015). The changes in flux values for most sources are $\sim 10 \%$, well within the nominal flux uncertainties of $\sim 30 \%$.

\subsubsection{DIGIT}

The photometric data used to construct the SEDs of DIGIT sources have similar wavelength coverage and sampling as that for HOPS sources. The 2MASS and Spitzer-IRAC, MIPS, and IRS data were compiled from those presented in Green et al. (2013b, and references therein). As for HOPS sources, the Spitzer IRS spectra have been rebinned to 12 flux values before integrating the SEDs. In addition, we obtained the PACS 70, 100 , and $160 \mu \mathrm{m}$ and SPIRE 250, 350, and $500 \mu \mathrm{m}$ data for the DIGIT sources from the Gould Belt Survey (e.g., André et al. 2010) and a few other Herschel KP and OT programs (Dunham 2010; Krause 2010; Stutz et al. 2010; Tobin 2010; Launhardt et al. 2013). The SPIRE photometry was extracted with DAOPhot within the Herschel Interactive Processing Environment (HIPE) using a top-hat annulus. In order to derive aperture extraction sizes, we used the Semi-Extended Source Corrector with HIPE. This routine matches the SPIRE spectral bands by varying the source size. We then used that source size as our annulus for extraction of SPIRE photometry. The aperture sizes used range from $22^{\prime \prime}-47^{\prime \prime}$. The same apertures were used to extract PACS photometry (for details, see Green et al. 2016, Y.-L. Yang et al. 2016, in preparation). We also made use of the ground-based submillimeter/millimeter photometry for the DIGIT sources presented in Green et al. (2013b).

\subsubsection{PBRs}

The photometry of the PBRs were obtained from the HOPS catalog (see Stutz et al. 2013; Furlan et al. 2016).

\subsection{Protostellar Properties}

We computed the bolometric luminosity, $L_{\mathrm{bol}}$, bolometric temperature, $T_{\text {bol }}$ and fractional submillimeter luminosity, $L_{\mathrm{bol}} / L_{\mathrm{smm}}$ of the protostars from their SEDs. $L_{\mathrm{bol}}$ was obtained by integrating under the observed SED over wavelength. We used trapezoidal integration for computing $L_{\text {bol }}$. The SEDs were extrapolated from the longest observed wavelength (in most cases, $850 \mu \mathrm{m})$ as $F_{\nu} \propto \nu^{2}$, before computing $L_{\mathrm{bol}}$. The bolometric temperature, defined as the temperature of a blackbody with the same mean frequency as the source SED, was computed from the mean frequency of the source SED, following the method of Myers \& Ladd (1993). The submillimeter luminosity, $L_{\mathrm{smm}}$ was computed by integrating under the SED longward of $350 \mu \mathrm{m}$. The protostellar parameters $L_{\mathrm{bol}}, T_{\mathrm{bol}}$, and $L_{\mathrm{bol}} / L_{\mathrm{smm}}$ are computed in a uniform way for the HOPS, DIGIT, and PBR sources in our sample and are listed in Table 1. For HOPS and PBR sources, which are in Orion, we used a distance of $420 \mathrm{pc}$ (Menten et al. 2007; Sandstrom et al. 2007; Kim et al. 2008) to compute the luminosities. For the DIGIT sources, the distances listed in Green et al. (2013b) were used. 

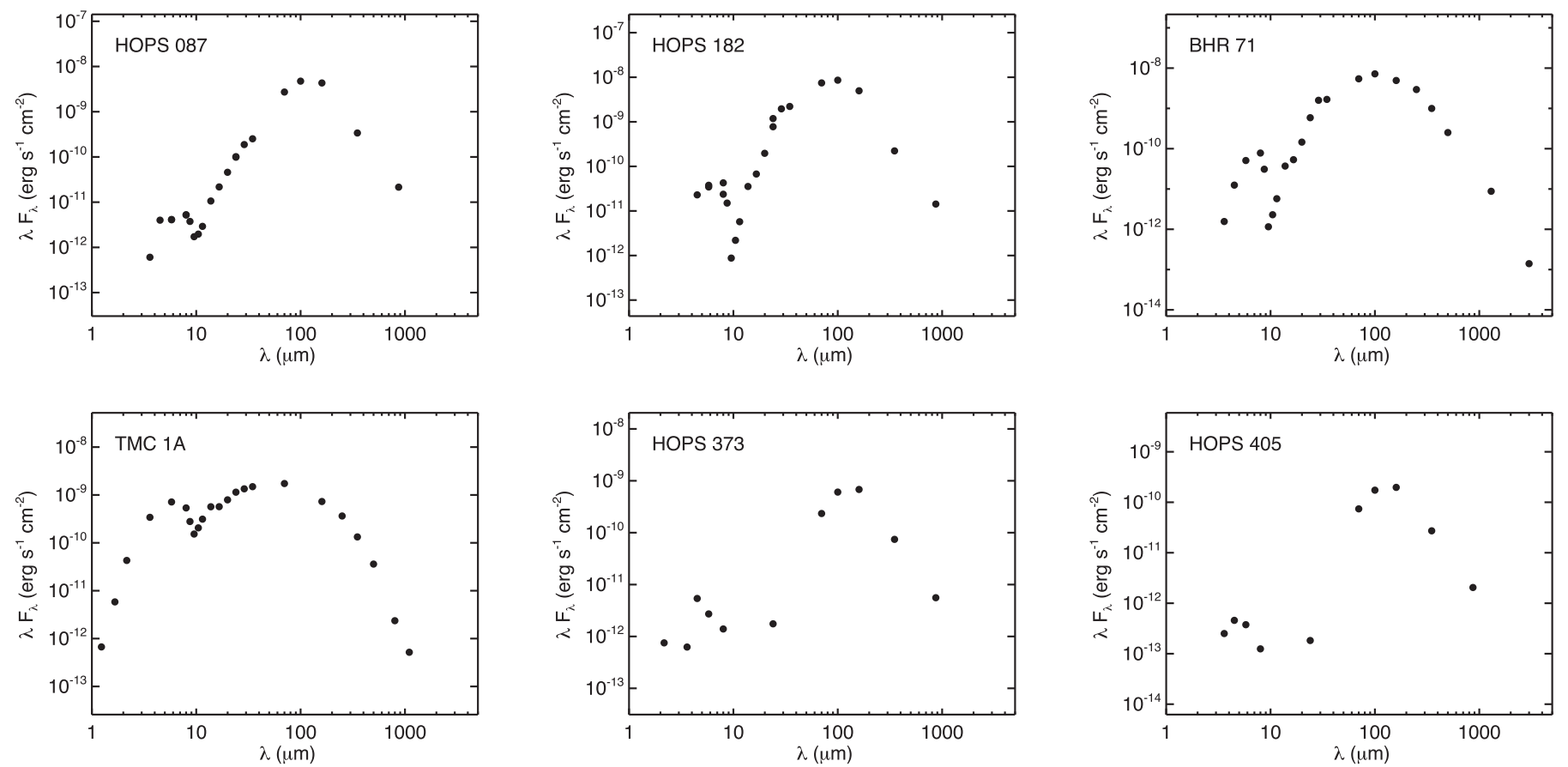

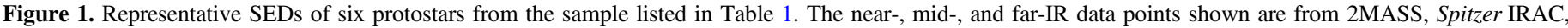

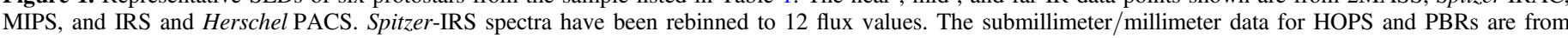

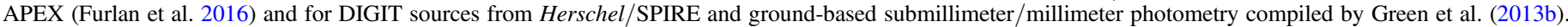

For the HOPS sources, the $L_{\text {bol }}$ values listed in Table 1 differ from that of Manoj et al. (2013) by $\sim 14 \%$ (median); the median difference in $T_{\mathrm{bol}}$ is $\sim 7 \%$. These changes can be attributed to the improved photometry used in constructing the observed SED, as described in Section 3.1.1. The $L_{\text {bol }}$ and $T_{\text {bol }}$ values of HOPS and PBR sources listed in Table 1 agree well with those presented in Furlan et al. (2016): for most sources they agree within 5\%-6\%. The major difference is for HOPS 369, which has been modeled as a double source comprising a diskdominated source and a protostar by Adams et al. (2012). We integrated the SED upward of $37 \mu \mathrm{m}$ to account only for the protostellar component (see Manoj et al. 2013), whereas Furlan et al. (2016) integrated under the entire SED to obtain $L_{\mathrm{bol}}$ and $T_{\text {bol }}$. For the DIGIT sources, the $L_{\mathrm{bol}}$ and $T_{\mathrm{bol}}$ values listed in Table 1 differ from those in Green et al. (2013b) by $\sim 15 \%$ (median). This is because we have used Herschel PACS photometry $(70,100$, and $160 \mu \mathrm{m})$ to sample the peak of the SED, while Green et al. (2013b) used PACS spectra, which typically have an absolute flux uncertainty of up to $\sim 30 \%$, in their SEDs. In summary, we have estimated the protostellar properties for HOPS/PBR and DIGIT sample uniformly, and these quantities agree well with previous estimates within $15 \%-20 \%$

As can be seen from Table 1, a key feature of our sample is the large range (more than three orders of magnitude) in $L_{\mathrm{bol}}$, from 0.1 to $275 L_{\odot}$. Also, the bolometric temperature, $T_{\text {bol }}$ ranges from 15 to $605 \mathrm{~K}$, indicating that our sample spans a wide range in the evolutionary sequence, from early to late protostellar phases.

\subsection{Far-infrared CO Data}

The PACS spectra of the HOPS sources used to measure CO line fluxes were presented in Manoj et al. (2013). The CO line fluxes were measured from the spectra extracted from the central spaxel of the rebinned cube, after applying the PSF loss correction (see Manoj et al. 2013). The far-IR CO luminosity, $L_{\mathrm{CO}}^{\mathrm{fir}}$, obtained by adding up the luminosities of all the $\mathrm{CO}$ lines detected with PACS, and the luminosity of the CO $(J=14 \rightarrow 13)$ line, $L_{\mathrm{CO}(\mathrm{J}=14-13)}$ for all the HOPS sources are listed in Table 1. As before, we used a distance of $420 \mathrm{pc}$ to Orion to compute the luminosities. For the DIGIT sources, the CO line fluxes were measured from the spectra of these sources presented in Green et al. (2016). The $L_{\mathrm{CO}}^{\mathrm{fir}}$ and $L_{\mathrm{CO}(\mathrm{J}=14-13)}$ for DIGIT sources are shown in Table 1. The distances to the DIGIT sources were taken from Green et al. (2013b). The farIR PACS spectra of PBRs were obtained as part of the Herschel OT program OT2_jtobin_2. The details of observations and data reductions can be found in Tobin et al. (2016). The CO luminosities of the eight PBRs in our sample are listed in Table 1.

\section{RESULTS}

\subsection{Far-IR CO Luminosity and $L_{b o l}$}

The total far-IR CO luminosity observed with PACS, $L_{\mathrm{CO}}^{\mathrm{fir}}$, is shown as a function of $L_{\text {bol }}$ in Figure 2(a) for HOPS, DIGIT, and PBR sources. One of the HOPS sources, HOPS 108, which is in the OMC-2 region (otherwise known as OMC-2 FIR 4) has the highest $L_{\mathrm{CO}}^{\text {fir }}$ among our sample. It also has the brightest line spectra of all the protostars in the HOPS sample (Manoj et al. 2013; Furlan et al. 2014). However, it has recently been shown that the intense line emission seen toward HOPS 108 is not associated with this protostar, but, instead originates in the terminal shock produced by the powerful jet driven by OMC-2 FIR 3 (HOPS 370; Gonzalez-Garcia et al. 2016). Therefore, we do not include HOPS 108 in further analysis. For seven sources, $L_{\mathrm{CO}}^{\mathrm{fir}}$ values are upper limits (downward arrows in Figure 2): no CO lines are detected in these sources. 

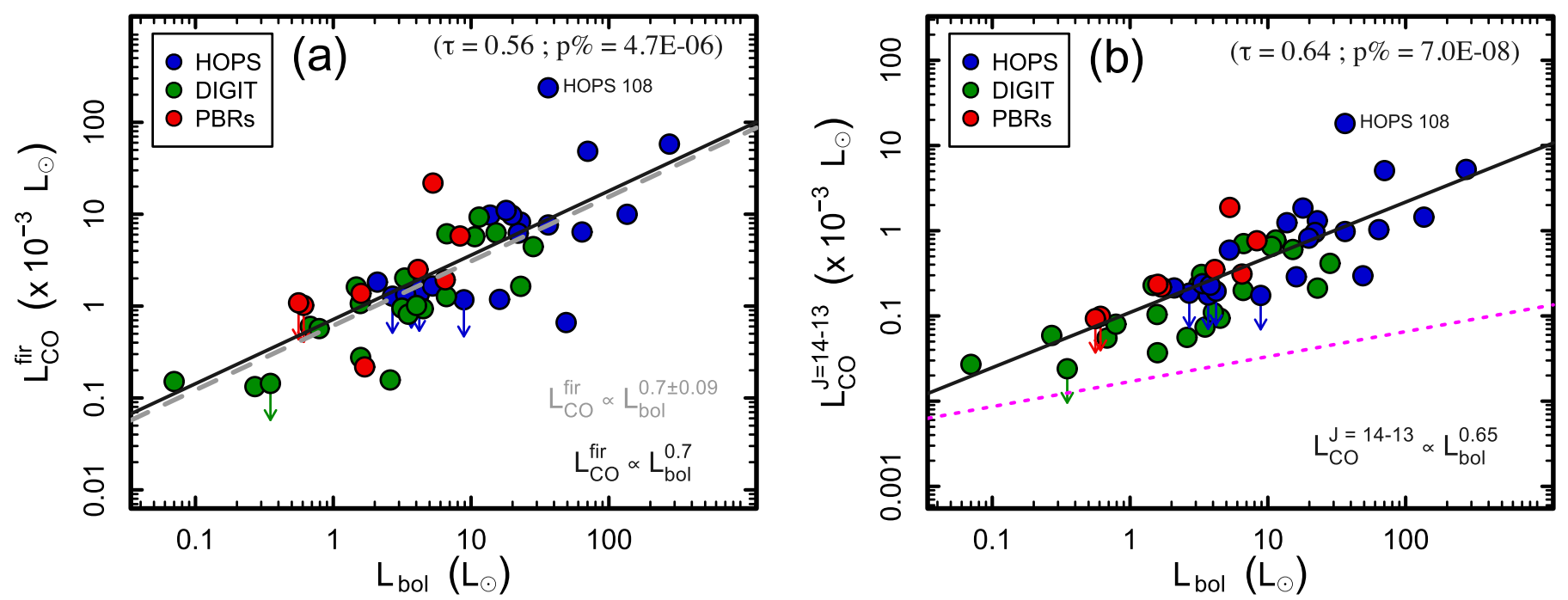

Figure 2. (a) FIR CO luminosity, with $L_{\mathrm{CO}}^{\text {fir }}$ as a function of $L_{\mathrm{bol}}$. The HOPS and DIGIT protostars are shown as blue and green solid circles, respectively, and the PBRs are shown in red. The downward arrows indicate upper limits in $L_{\mathrm{CO}}^{\mathrm{fir}}$. The gray dashed line corresponds to the best least-square fit to the detections only. The black solid line is the Akritas-Thiel-Sen (ATS) line including the censored points. (b) Luminosity of the $\mathrm{CO}(J=14 \rightarrow 13)$ line, $L_{\mathrm{CO}(\mathrm{J}=14-13)}$ as a function of $L_{\mathrm{bol}}$. The dashed magenta line indicates the sensitivity of our PACS CO observations converted into luminosity. It has the form $L_{\mathrm{CO}(\mathrm{J}=14-13)} \propto L_{\mathrm{bol}}^{0.3}$. The black line is the ATS line. Generalized Kendall's $\tau$ values and associated probabilities are shown in both panels.

Table 2

Correlation Coefficients (Kendall's $\tau$ ) and Associated Probabilities ${ }^{\mathrm{a}}$

\begin{tabular}{|c|c|c|c|c|c|c|}
\hline & $T_{\text {bol }}$ & $L_{\mathrm{bol}} / L_{\mathrm{smm}}$ & $L_{\mathrm{CO}}^{\mathrm{fir}}$ & $L_{\mathrm{CO}(\mathrm{J}=14-13)}$ & $L_{\mathrm{CO}}^{\mathrm{fir}} / L_{\mathrm{bol}} 0.7$ & $L_{\mathrm{CO}(\mathrm{J}=14-13)} / L_{\mathrm{bol}}^{0.65}$ \\
\hline$L_{\text {bol }}$ & $0.1(31 \%)$ & $\mathbf{0 . 4}\left(\mathbf{9} \times 10^{-4} \%\right)$ & $\mathbf{0 . 5 6}\left(\mathbf{5} \times 10^{-6} \%\right)$ & $0.64\left(7 \times 10^{-8} \%\right)$ & $\ldots$ & $\ldots$ \\
\hline$T_{\mathrm{bol}}$ & $\ldots$ & $\ldots$ & $-0.1(29 \%)$ & $\ldots$ & $-0.3(0.2 \%)$ & $-0.3(0.2 \%)$ \\
\hline$L_{\mathrm{bol}} / L_{\mathrm{smm}}$ & $\cdots$ & $\cdots$ & $0.2(3.1 \%)$ & $\cdots$ & $\ldots$ & $\ldots$ \\
\hline
\end{tabular}

Note.

${ }^{a}$ For correlations involving $L_{\mathrm{CO}}^{\mathrm{fir}}$ and $L_{\mathrm{CO}(\mathrm{J}=14-13)}$, which have upper limit points, generalized Kendall's tau and associated probablity are listed. Correlation coefficient and probabilities shown in bold are statistically highly significant.

Figure 2(a) shows that $L_{\mathrm{CO}}^{\mathrm{fir}}$ is strongly correlated with $L_{\mathrm{bol}}$ over three orders of magnitude in both quantities. For the detections (non-upper limits), the Pearson's product-moment correlation between $\log \left(L_{\mathrm{CO}}^{\text {fir }}\right)$ and $\log \left(L_{\mathrm{bol}}\right)$ is 0.77 and the associated probability that these two quantities are uncorrelated is $\ll 10^{-6} \%$. Kendall's rank correlation coefficient, $\tau$, between $L_{\mathrm{CO}}^{\mathrm{fir}}$ and $L_{\mathrm{bol}}$ is 0.56 and the associated probability $p<10^{-5 \%}$ and the Spearman's $\rho=0.74$ and probability $p<10^{-6 \%}$, indicating that the correlation between far-IR $L_{\mathrm{CO}}^{\mathrm{fir}}$ and $L_{\mathrm{bol}}$ is strong. The functional dependence of $L_{\mathrm{CO}}^{\mathrm{fir}}$ on $L_{\mathrm{bol}}$ obtained using an ordinary least-square fit ${ }^{10}$ (excluding the upper limits in $L_{\mathrm{CO}}^{\text {fir }}$ ) is found to be $L_{\mathrm{CO}}^{\text {fir }} \propto L_{\mathrm{bol}}^{0.7 \pm 0.09}$.

We also computed the correlation coefficient and the functional dependence of $L_{\mathrm{CO}}^{\mathrm{fir}}$ on $L_{\mathrm{bol}}$ including the censored data points (upper limits in $L_{\mathrm{CO}}^{\text {fir }}$ ). For this, we used the AkritasThiel-Sen (ATS) regression method, which is the extension of the Thiel-Sen regression method (Sen 1968) for censored data (Akritas et al. 1995; Feigelson \& Babu 2012). The Thiel-Sen regression obtains a slope that is the median of the $n(n+1) / 2$ slopes of lines defined by all pairs of data points and can be formulated in terms of Kendall's tau rank correlation coefficient (Sen 1968). For censored data, pairwise slopes involving censored data points lie in a range of possible values,

\footnotetext{
${ }^{10}$ We used the STATS package in the R statistical software system (R Core Team 2015) to carry out the statistical tests and least-square fit.
}

and the ATS method estimates a distribution function of slopes with these interval-censored values. The median of this distribution becomes a slope estimator for the censored data. The ATS method also provides the generalized Kendall's tau rank correlation coefficient (Brown et al. 1974) for data including censored points (see Akritas et al. 1995; Feigelson \& Babu 2012).

The generalized Kendall's $\tau$ for the correlation ${ }^{11}$ between $L_{\mathrm{CO}}^{\mathrm{fir}}$ and $L_{\mathrm{bol}}$ is 0.56 and the associated probability is 5.0 $\times 10^{-6} \%$ indicating that the correlation is statistically highly significant. The correlation coefficients and the associated probabilities for the correlations between various quantities are shown in Table 2. The best-fit ATS line, which provides the functional dependence of $L_{\mathrm{CO}}^{\mathrm{fir}}$ on $L_{\mathrm{bol}}$, including the censored data, is shown in Figure 2(a) and has the form

$$
\log L_{\mathrm{CO}}^{\mathrm{fir}}=-3.2+0.7 \log L_{\mathrm{bol}}
$$

which can be written as

$$
L_{\mathrm{CO}}^{\mathrm{fir}}=6 \times 10^{-4} L_{\mathrm{bol}}^{0.7}
$$

\footnotetext{
${ }^{11}$ Throughout the paper, we have used the NADA package (Lee 2013) in R (R Core Team 2015) to compute the best-fit ATS line and the generalized Kendall's tau rank correlation coefficient.
} 

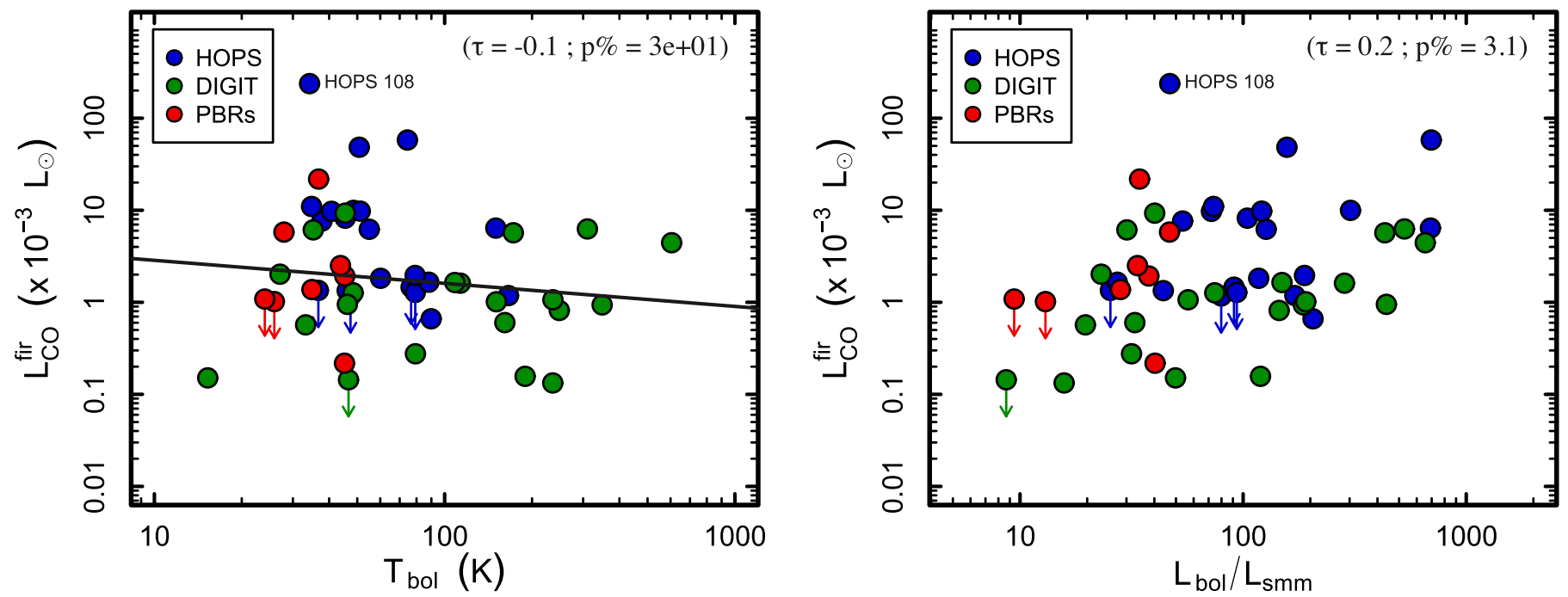

Figure 3. FIR CO luminosity as a function of evolutionary indicators $T_{\mathrm{bol}}$ and $L_{\mathrm{bol}} / L_{\mathrm{smm}}$. The HOPS and DIGIT protostars shown as blue and green solid circles, respectively, and the PBRs are shown in red. The downward arrows indicate upper limits in $L_{\mathrm{CO}}^{\mathrm{fir}}$. The black line is the ATS line. Generalized Kendall's $\tau$ values and associated probabilities are shown in both panels.

or equivalently as

$$
L_{\mathrm{CO}}^{\mathrm{fir}} / L_{\mathrm{bol}}=6 \times 10^{-4} L_{\mathrm{bol}}^{-0.3} .
$$

The far-IR CO luminosity is only a small fraction of the protostellar luminosity $L_{\mathrm{bol}}$ and the ratio $L_{\mathrm{CO}}^{\mathrm{fir}} / L_{\mathrm{bol}}$ drops toward more luminous sources. $L_{\mathrm{CO}}^{\mathrm{fir}} / L_{\mathrm{bol}}$ ranges from $0.4 \%-$ $0.001 \%$ for the protostars in our sample. Median values of $L_{\mathrm{CO}}^{\mathrm{fir}} / L_{\mathrm{bol}}$, as a function of $L_{\mathrm{bol}}$, range from $0.1 \%$ for sources with $L_{\text {bol }}<1 L_{\odot}$ to $0.02 \%$ for $L_{\text {bol }}>100 L_{\odot}$.

\subsubsection{Scatter in the $L_{c o}^{f i r}-L_{b o l}$ Relation}

While the correlation between $L_{\mathrm{CO}}^{\text {fir }}$ and $L_{\mathrm{bol}}$ is strong and reasonably tight over three orders of magnitude in both the quantities, there is some spread in the observed $L_{\mathrm{CO}}^{\mathrm{fir}}$ values for a given $L_{\mathrm{bol}}$. The mean absolute deviation from the best-fit line in Figure 2 is about a factor of two in $L_{\mathrm{CO}}^{\mathrm{fir}}$ and the maximum deviation, a factor of 16 lower, is detected for HOPS 84, which has an $L_{\mathrm{bol}}$ of $49 L_{\odot}$, but only three detected CO lines. Part of this scatter is because not all CO lines in the PACS range are detected for all of the sources. $L_{\mathrm{CO}}^{\text {fir }}$ is computed by adding up the luminosities of all the detected lines, and, therefore, sources in which more $\mathrm{CO}$ lines are detected will have significantly higher $L_{\mathrm{CO}}^{\text {fir }}$ compared to those in which very few lines are detected. The scatter produced by this effect can be minimized if the luminosity of a single $\mathrm{CO}$ line is plotted. To illustrate this, we show $\mathrm{CO} \quad(J=14 \rightarrow 13)$ line luminosity, $L_{\mathrm{CO}(\mathrm{J}=14-13)}$, as a function of $L_{\mathrm{bol}}$ in Figure 2(b). The correlation between $L_{\mathrm{CO}(\mathrm{J}=14-13)}$ and $L_{\mathrm{bol}}$ is stronger than that found for $L_{\mathrm{CO}}^{\mathrm{fir}}$ : the generalized Kendall's $\tau$ (including the censored data points) for the correlation is 0.64 and the associated probability is $7.0 \times 10^{-8 \%}$. The best-fit ATS line shown in Figure 2(b) has the form $L_{\mathrm{CO}(\mathrm{J}=14-13)} \propto L_{\mathrm{bol}}^{0.65}$. The correlation between $L_{\mathrm{CO}(\mathrm{J}=14-13)}$ and $L_{\mathrm{bol}}$ is also tighter: the mean absolute deviation from the best-fit line is only about a factor of 1.6 in $L_{\mathrm{CO}(\mathrm{J}=14-13)}$ and the maximum deviation from the fit is only about a factor of six. Thus the true dispersion in $L_{\mathrm{CO}}^{\text {fir }}-L_{\mathrm{bol}}$ relation is only a factor of few. To summarize, farIR CO line luminosities exhibit a strong and tight correlation with $L_{\text {bol }}$ over three orders of magnitude, with an average dispersion of a factor of a few.

\subsubsection{Sensitivity and the $L_{c o}^{f i r}-L_{b o l}$ Relation}

The observed $1 \sigma$ sensitivities of CO line fluxes for the HOPS and DIGIT sample are very similar. For example, the median $\mathrm{CO}(J=14 \rightarrow 13)$ line sensitivity for the HOPS sample is 1.1 $\times 10^{-17} \mathrm{Wm}^{-2}$, while that for the DIGIT sample is 1.3 $\times 10^{-17} \mathrm{Wm}^{-2}$. However, the HOPS and the PBR sources are in Orion, and are at the same distance $(420 \mathrm{pc})$, while the DIGIT sources are at various distances ranging from 106 to $325 \mathrm{pc}$. This will result in different sensitivities of $\mathrm{CO}$ line luminosities for the HOPS (and PBRs) and the DIGIT sources. The median sensitivity of $\mathrm{CO}(J=14 \rightarrow 13)$ line luminosity for the DIGIT sources is about five times lower than that for HOPS and PBR sources. In addition, the DIGIT sample preferentially has lower $L_{\text {bol }}$ sources compared to HOPS and PBR samples. The resulting dependence of the sensitivity on $L_{\text {bol }}$ may result in a spurious correlation if there are many nondetections and the detections are just above the sensitivity limit. We show the typical observed sensitivity for the $\mathrm{CO}$ $(J=14 \rightarrow 13)$ line as a function of $L_{\text {bol }}$ in Figure 2(b). The observed $L_{\mathrm{CO}(\mathrm{J}=14-13)}$ are significantly above the sensitivity for most $L_{\mathrm{bol}}$. The sensitivity and the sample bias can at best produce an $L_{\mathrm{bol}}$ dependence of $L_{\mathrm{CO}(\mathrm{J}=14-13)} \propto L_{\mathrm{bol}}^{0.3}$ (the dashed magenta line in Figure 2(b)). Thus the correlation between far-IR CO luminosities and $L_{\text {bol }}$ is robust.

\section{2. $L_{c o}^{f i r}$ and Evolutionary Indicators}

The bolometric temperature, $T_{\mathrm{bol}}$, and the fractional submillimeter luminosity, $L_{\mathrm{smm}} / L_{\mathrm{bol}}$, are the two commonly used observational tracers of protostellar evolution (e.g., André et al. 1993, 2000; Chen et al. 1995; Evans et al. 2009). In the following, instead of $L_{\mathrm{smm}} / L_{\mathrm{bol}}$, we use $L_{\mathrm{bol}} / L_{\mathrm{smm}}$ so that both $T_{\text {bol }}$ and $L_{\mathrm{bol}} / L_{\mathrm{smm}}$ increase as the protostellar system ages: i.e., more evolved protostars will have larger values of $T_{\text {bol }}$ and $L_{\mathrm{bol}} / L_{\mathrm{smm}}$. $L_{\mathrm{CO}}^{\mathrm{fir}}$ as a function of $T_{\mathrm{bol}}$ and $L_{\mathrm{bol}} / L_{\mathrm{smm}}$ is shown in Figure 3. For the sample of protostars presented here, far-IR $L_{\mathrm{CO}}^{\mathrm{fir}}$ does not show any statistically significant 

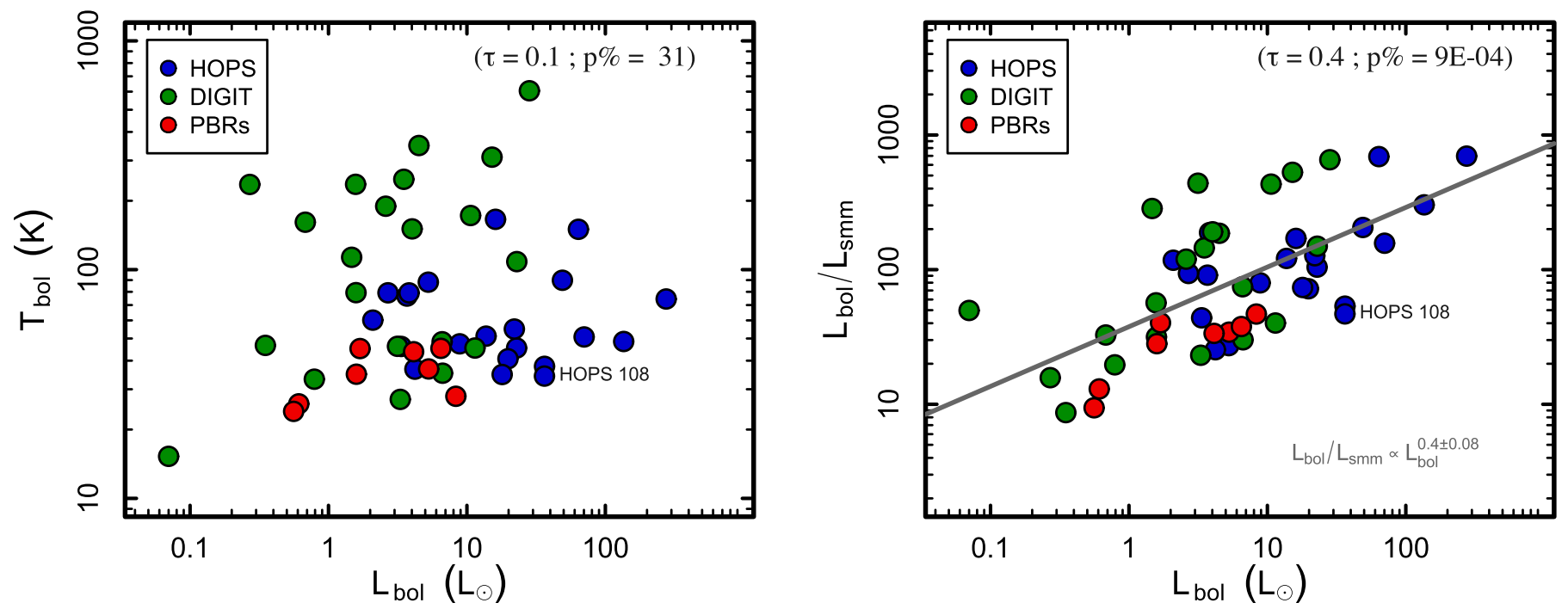

Figure 4. $T_{\mathrm{bol}}$ and $L_{\mathrm{bol}} / L_{\mathrm{smm}}$ as functions of $L_{\mathrm{bol}}$. The HOPS and DIGIT protostars are shown as blue and green solid circles, respectively, and the PBRs are shown in red. The gray solid line is the least square fit. Kendall's $\tau$ values and associated probabilities are shown in both panels.

correlation with the evolutionary indicators $T_{\mathrm{bol}}$ and $L_{\mathrm{bol}} / L_{\mathrm{smm}}$. The generalized Kendall's $\tau$ (including upper limits) for the correlation between $L_{\mathrm{CO}}^{\mathrm{fir}}$ and $T_{\mathrm{bol}}$ is -0.1 and the probability that these two quantities are uncorrelated is $29 \%$. $L_{\mathrm{CO}}^{\text {fir }}$, on the other hand, shows a marginal positive correlation $(\lesssim 2 \sigma)$ with $L_{\mathrm{bol}} / L_{\mathrm{smm}}(\tau=0.22$ and $p=3.1 \%)$, which seems to suggest that $L_{\mathrm{CO}}^{\mathrm{fir}}$ increases as the protostar ages, contrary to what is generally expected. This may result from the strong $L_{\mathrm{bol}}$ dependence of $L_{\mathrm{bol}} / L_{\mathrm{smm}}$, as can be seen from Figure 4 . While $T_{\mathrm{bol}}$ is independent of $L_{\mathrm{bol}}(\tau=0.1 ; p=30 \%)$, $L_{\mathrm{bol}} / L_{\mathrm{smm}}$ is strongly correlated with $L_{\mathrm{bol}}(\tau=0.4 ; p=9$ $\left.\times 10^{-4} \%\right)$ and has a functional dependence of the form $L_{\mathrm{bol}}{ }^{0.4}$. The apparent positive correlation between $L_{\mathrm{CO}}^{\mathrm{fir}}$ and $L_{\mathrm{bol}} / L_{\mathrm{smm}}$ seen in Figure 3 is caused by the $L_{\mathrm{bol}}$ dependence of both quantities.

Although both $T_{\mathrm{bol}}$ and $L_{\mathrm{bol}} / L_{\mathrm{smm}}$ are measures of system age and both are derived from the observed SEDs, $T_{\text {bol }}$ does not show any $L_{\mathrm{bol}}$ dependence, while $L_{\mathrm{bol}} / L_{\mathrm{smm}}$ shows a convincing correlation with $L_{\mathrm{bol}}$. This is because $L_{\mathrm{smm}}$ for our sample sources scales as $L_{\mathrm{bol}}^{0.6}$ and not linearly with $L_{\mathrm{bol}}$ as one would expect. One possible explanation for this is that the outer regions of the protostellar envelopes are also heated externally by the interstellar radiation field, in addition to the heating from the central star. The relative contribution of external heating to $L_{\mathrm{smm}}$ will be higher in low $L_{\mathrm{bol}}$ sources, thus producing a sublinear dependence of $L_{\mathrm{smm}}$ on $L_{\mathrm{bol}}$. Additionally, the $L_{\mathrm{bol}}$ is highly sensitive to inclination angle (see Furlan et al. 2016), increasing significantly going from an edge-on to a face-on viewing geometry. $L_{\mathrm{smm}}$, on the other hand, is relatively insensitive to the changes in the viewing angle, resulting in a flatter dependence of $L_{\mathrm{smm}}$ on $L_{\mathrm{bol}}$.

It is possible that the strong $L_{\mathrm{bol}}$ dependence of $L_{\mathrm{CO}}^{\mathrm{fir}}$ is masking evolutionary trends in $L_{\mathrm{CO}}^{\mathrm{fir}}$. In order to check this and to emphasize evolutionary trends in $L_{\mathrm{CO}}^{\mathrm{fir}}$, we removed the $L_{\mathrm{bol}}$ dependence of $L_{\mathrm{CO}}^{\mathrm{fir}}$ and $L_{\mathrm{bol}} / L_{\mathrm{smm}}$ by normalizing them with the functional form of their $L_{\mathrm{bol}}$ dependence. We then searched for correlations between these normalized quantities as shown in Figure 5. The normalized far-IR CO luminosity, $L_{\mathrm{CO}}^{\mathrm{fir}} / L_{\mathrm{bol}}^{0.7}$, which is independent of $L_{\text {bol }}$, shows a weak $(\sim 3 \sigma)$ negative correlation with $T_{\text {bol }}$, indicating that far-IR CO luminosity drops as the protostellar system evolves. The generalized Kendall $\tau$ for the correlation is -0.3 and the associated probability is $0.2 \%$. The ATS non-parametric line fit, including the upper limits, gives a $T_{\text {bol }}$ dependence of the form

$$
L_{\mathrm{CO}}^{\mathrm{fir}} \propto L_{\mathrm{bol}}^{0.7} T_{\mathrm{bol}}^{-0.5} .
$$

However, far-IR $L_{\mathrm{CO}}^{\text {fir }}$ is not correlated with $L_{\mathrm{bol}} / L_{\mathrm{smm}}$, even after removing the $L_{\mathrm{bol}}$ dependence in both the quantities as shown in Figure 5. The generalized Kendall $\tau$ for the correlation is -0.2 and the associated probability is $11 \%$, indicating that these quantities are uncorrelated.

Individual CO line luminosities also display similar behavior with protostellar evolutionary indicators. In Figure 6, we show $L_{\mathrm{CO}(\mathrm{J}=14-13)}$ normalized to its $L_{\mathrm{bol}}$ dependence as a function of $T_{\mathrm{bol}}$ and $L_{\mathrm{bol}} / L_{\mathrm{smm}}$ normalized to its $L_{\mathrm{bol}}$ dependence. $L_{\mathrm{CO}(\mathrm{J}=14-13)} / L_{\mathrm{bol}}^{0.65}$ shows a weak or no correlation with protostellar evolutionary indicators.

\section{DISCUSSION}

\subsection{Mass Accretion-ejection Connection in Protostars}

The far-IR CO luminosity, $L_{\mathrm{CO}}^{\text {fir }}$ is the total luminosity of the high-excitation CO lines in the far-IR (50-200 $\mu \mathrm{m})$ observed with PACS on board Herschel. Manoj et al. (2013) have demonstrated that the far-IR CO lines observed toward lowmass protostars arise in hot $(T \gtrsim 2000 \mathrm{~K})$ gas heated in outflow shocks, a conclusion supported by various other studies with Herschel (e.g., Karska et al. 2013; Matuszak et al. 2015). The cooling timescale for the postshock gas at $T \gtrsim 2000 \mathrm{~K}$ and $n\left(\mathrm{H}_{2}\right) \sim 10^{4}-10^{6} \mathrm{~cm}^{-3}$ is $<100$ years, which is significantly shorter than the protostellar lifetimes ( $0.5 \mathrm{Myr}$; Dunham et al. 2014b). The far-IR CO emission from protostars traces the gas that is currently being shocked by jets/outflows. $L_{\mathrm{CO}}^{\mathrm{fir}}$, then, must be proportional to the total cooling radiation from the shocked gas which, in turn, is proportional to the energy dissipated by jets/outflows. Although other atomic and molecular species such as [O I], water, and $\mathrm{OH}$ can contribute to the cooling in the far-IR, Herschel studies of protostars have shown that $L_{\mathrm{CO}}^{\mathrm{fir}}$ is $\sim 30 \%-40 \%$ of the total far-IR-cooling, and, 

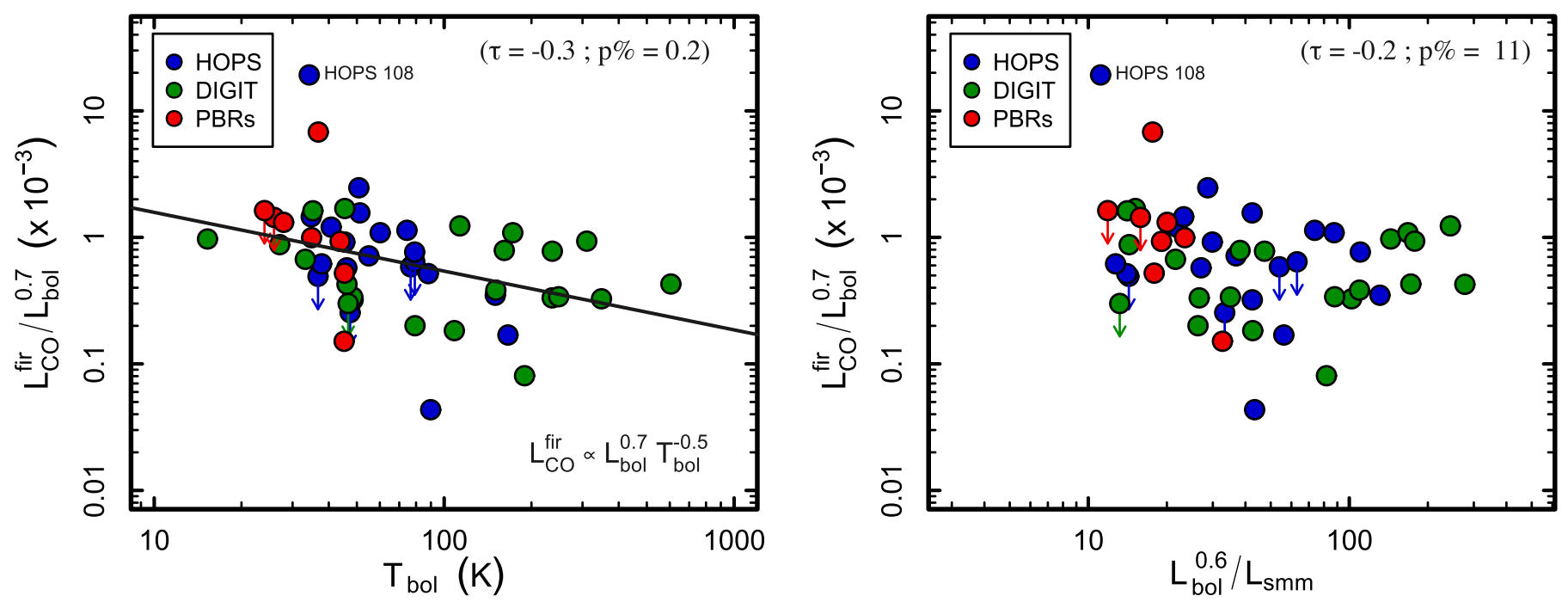

Figure 5. Normalized $L_{\mathrm{CO}}^{\text {fir }}$ as a function of $T_{\mathrm{bol}}$ and $L_{\mathrm{bol}} / L_{\mathrm{smm}}$. The HOPS and DIGIT protostars shown as blue and green solid circles, respectively, and the PBRs are shown in red. The downward arrows indicate upper limits on $L_{\mathrm{CO}}^{\text {fir }}$. The black line is the ATS line. Generalized Kendall's $\tau$ values and associated probabilities are shown in both panels.

in addition, is proportional to the total far-IR luminosity (Karska et al. 2013; Lee et al. 2014a; P. Manoj et al. 2016, in preparation). Thus, $L_{\mathrm{CO}}^{\text {fir }}$ can be taken as a lower limit to the mechanical luminosity $\left(L_{\text {mech }}\right)$ of the jets/outflows dissipated in shocks over a timescale of $<100$ years. In addition, the observed $L_{\mathrm{CO}}^{\text {fir }}$ only includes $\mathrm{CO}$ emission from a compact region around the base of the jet/outflow. For Orion sources (HOPS and PBRs) the spatial extent of the observed CO emission is within a radius of $\sim 2000$ au from the protostar (see Manoj et al. 2013). For DIGIT sources, which are closer, the spatial extent probed is even smaller. The dynamical timescale of such a flow is only $100-200$ years for flow velocities of 50 $-100 \mathrm{~km} \mathrm{~s}^{-1}$. Thus both the cooling timescale and the dynamical timescale of the observed flow are extremely short, and only a small fraction of the protostellar lifetime. $L_{\mathrm{CO}}^{\mathrm{fir}}$, therefore, must be proportional to the instantaneous (smoothed over $\sim 100$ years) $L_{\text {mech }}=1 / 2 \dot{M}_{\text {out }} v_{\text {out }}^{2}$, which ranges over three orders of magnitude for our sample. The jet/outflow velocity, $v_{\text {out }}$, in protostars are likely to differ by a factor of a few at the most, and the large range in $L_{\text {mech }}\left(L_{\mathrm{CO}}^{\text {fir }}\right)$ is primarily due to the mass-loss rate, $\dot{M}_{\text {out }}$.

The protostellar luminosity, $L_{\mathrm{bol}}$ is given by

$$
L_{\mathrm{bol}}=L_{\mathrm{phot}}+L_{\mathrm{acc}} \text {, }
$$

where $L_{\text {phot }}$ is the photospheric luminosity generated by gravitational contraction and deuterium burning and $L_{\text {acc }}$ is the luminosity released from the accretion of material from disk onto the protostars, which is given by

$$
L_{\mathrm{acc}}=\eta \frac{G M(t) \dot{M}_{\mathrm{acc}}}{r}
$$

where $\eta$ is the fraction of energy radiated away in the accretion shock, $M(t)$ is the instantaneous protostellar mass, and $\dot{M}_{\text {acc }}$ is the accretion rate onto the protostar. The dominant contribution to $L_{\mathrm{bol}}$ is from $L_{\mathrm{acc}}$ as the contribution from $L_{\mathrm{phot}}$ becomes important only at late times during protostellar evolution (e.g., Tobin et al. 2012). The observed points used to construct the SED from which $L_{\mathrm{bol}}$ is computed were measured within a time span of $\sim 20-30$ years. Thus $L_{\mathrm{bol}}$ is a measure of instantaneous (smoothed over $\sim 30 \mathrm{yr}$ ) accretion luminosity, $L_{\mathrm{acc}}$, which depends on both $M(t)$ and $\dot{M}_{\text {acc }}$, but the large range (three orders of magnitude) in $L_{\mathrm{bol}}$ displayed by our sample sources is primarly driven by $\dot{M}_{\mathrm{acc}}$. Therefore, $L_{\mathrm{bol}}$ should scale with accretion rate, $\dot{M}_{\text {acc }}$, onto the protostar and the correlation seen in Figure 2 between $L_{\mathrm{CO}}^{\text {fir }}$ and $L_{\mathrm{bol}}$ is most likely the result of instantaneous $\dot{M}_{\text {out }}$ tracking instantaneous $\dot{M}_{\text {acc }}$. Most jet launching mechanisms proposed for protostars predict such a tight correlation between $\dot{M}_{\text {out }}$ and $\dot{M}_{\text {acc }}$ (e.g., Pelletier \& Pudritz 1992; Wardle \& Koenigl 1993; Shu et al. 1994)

The $L_{\mathrm{CO}}^{\text {fir }}-L_{\text {bol }}$ relation also shows an intrinsic dispersion, as demonstrated in Section 4.1.1. Various factors could produce such scatter. Although, $L_{\mathrm{CO}}^{\text {fir }}$ primarily tracks $\dot{M}_{\text {out }}$, it also depends on the flow velocity. $L_{\mathrm{CO}}^{\mathrm{fir}}$ could also depend on the properties of the immediate environment around protostars, such as envelope density and ambient gas density. The Orion sources (HOPS and PBRs) in our sample are from different and diverse regions within the Orion A (e.g., L1641, OMC2/3) and Orion B cloud. The DIGIT sources are from various different star-forming regions (Green et al. 2013b, 2016). In addition, $L_{\text {bol }}$ has a strong inclination angle dependence, while $L_{\mathrm{CO}}^{\text {fir }}$, which represents optically thin $\mathrm{CO}$ emission, is less likely to be affected by viewing geometry. All of these factors could introduce scatter in the $L_{\mathrm{CO}}^{\mathrm{fir}}-L_{\mathrm{bol}}$ relation. Despite this, the observed dispersion in the $L_{\mathrm{CO}}^{\mathrm{fir}}-L_{\text {bol }}$ relation is quite small: it is only a factor of a few compared to the range (three orders of magnitudes) over which the correlation holds. This is possibly because both $L_{\text {bol }}$ and $L_{\mathrm{CO}}^{\text {fir }}$ track $\dot{M}_{\text {acc }}$ and $\dot{M}_{\text {out }}$ smoothed over short and somewhat similar timescales, and, therefore, even if protostellar accretion is episodic, likely measure the same mass-accretion/ejection event. Thus, variations in $L_{\mathrm{bol}}$ are closely tracked by $L_{\mathrm{CO}}^{\mathrm{fir}}$, resulting in a strong correlation between the two quantities and a small dispersion.

\subsection{Evolution of Mass Accretion/Ejection in Protostars}

While the observed $L_{\mathrm{CO}}^{\text {fir }}$ of protostars is tightly correlated with their luminosity, $L_{\mathrm{bol}}$, it shows no, or at best a weak, correlation with evolutionary indicators such as $T_{\text {bol }}$ and 

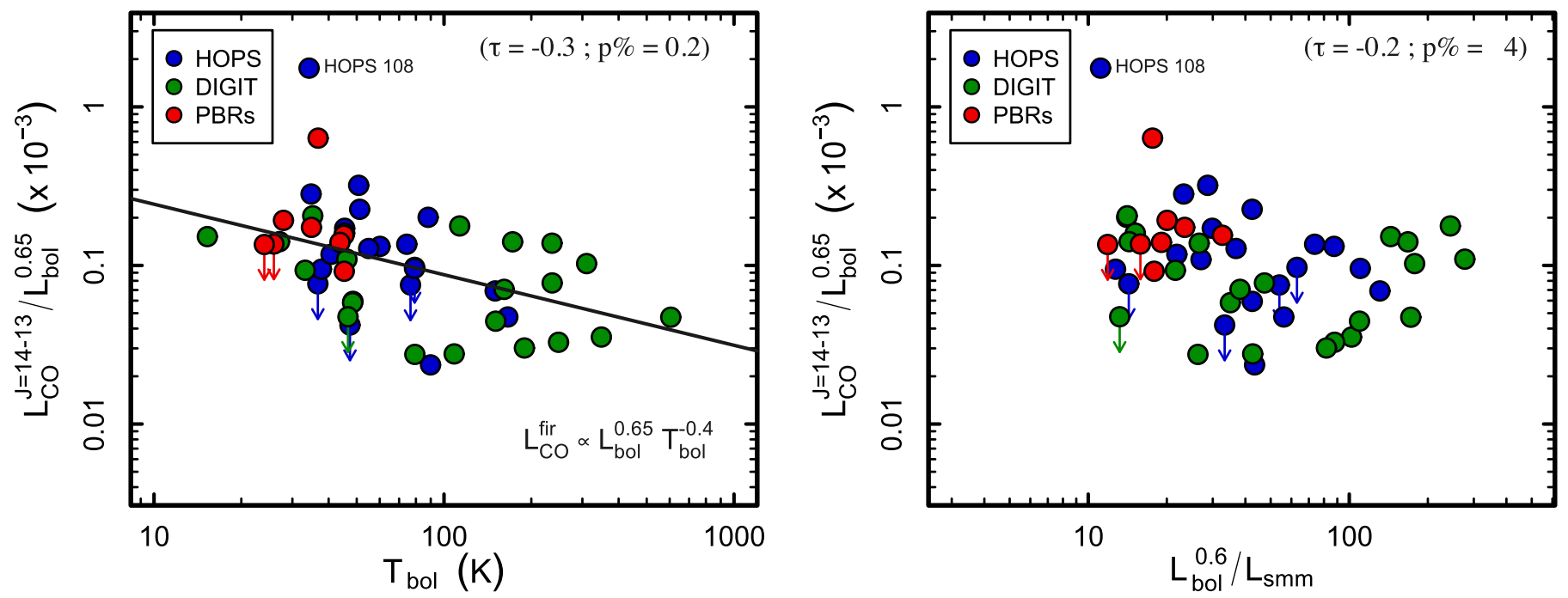

Figure 6. Normalized $L_{\mathrm{CO}(\mathrm{J}=14-13)}$ as a function of $T_{\mathrm{bol}}$ and $L_{\mathrm{bol}} / L_{\mathrm{smm}}$. The HOPS and DIGIT protostars shown as blue and green solid circles, respectively, and the PBRs are shown in red. The downward arrows indicate upper limits on $L_{\mathrm{CO}}^{\mathrm{fir}}$. The black line is the ATS line. Generalized Kendall's $\tau$ values and associated probabilities are shown in both panels.

$L_{\mathrm{bol}} / L_{\mathrm{smm}}$. As argued in Section 5.1, the tight correlation found between $L_{\mathrm{CO}}^{\text {fir }}$ and $L_{\mathrm{bol}}$ is primarily driven by instantaneous $\dot{M}_{\text {out }}$ closely tracking instantaneous $\dot{M}_{\text {acc }}$. Such a correlation is expected irrespective of the accretion history of protostars and is consistent with both episodic accretion and steady accretion scenarios. However, we find that $L_{\mathrm{CO}}^{\mathrm{fir}}$, which is proportional to instantaneous $\dot{M}_{\text {out }}$, shows only a marginal correlation with $T_{\text {bol }}$ and no correlation with $L_{\mathrm{bol}} / L_{\mathrm{smm}}$. If the accretion is steady, for example, with $\dot{M}_{\text {acc }}$ monotonically declining with the system age, one would expect a tight correlation between $L_{\mathrm{CO}}^{\mathrm{fir}}$ and the evolutionary indicators $T_{\mathrm{bol}}$ and $L_{\mathrm{bol}} / L_{\mathrm{smm}}$. On the other hand, if accretion were episodic, the instantaneous $\dot{M}_{\text {acc }}$ or $\dot{M}_{\text {out }}$ need not have a simple monotonic relation with system age. The lack of a strong correlation between $L_{\mathrm{CO}}^{\mathrm{fir}}$ and these evolutionary indicators suggests that accretion and jet/outflow activity in protostars are likely episodic. Moreover, if mass accretion is episodic, then $T_{\mathrm{bol}}$ and $L_{\mathrm{bol}} / L_{\mathrm{smm}}$ are not reliable indicators of the physical evolutionary stage of the protostars (Dunham et al. 2010). Thus our results are consistent with the mass accretion/ejection in protostars being episodic and do not support a steadily declining $\dot{M}_{\text {acc }}$ during the protostellar phase.

\subsection{Comparison with (sub)millimeter CO Emission from Outflows}

As pointed out in Section 1, observations of low- $J\left(J_{\text {up }} \leqslant 3\right)$ $\mathrm{CO}$ lines of the molecular outflows from protostars have shown that the mechanical luminosity, $L_{\mathrm{CO}}^{(\mathrm{sub}) \mathrm{mm}}$, and the momentum flux, $F_{\mathrm{CO}}$ of the molecular flow are correlated with $L_{\mathrm{bol}}$ (e.g., Lada 1985; Cabrit \& Bertout 1992; Bontemps et al. 1996; Wu et al. 2004; Curtis et al. 2010). $L_{\mathrm{CO}}^{(\mathrm{sub}) \mathrm{mm}}$ and $F_{\mathrm{CO}}$ are estimated from the total mass in the observed molecular flow and the characteristic velocity of the flow (see Cabrit \& Bertout 1992; Bachiller \& Tafalla 1999; Richer et al. 2000). The kinematical ages (apparent dynamical ages) of the observed molecular flows in low- $J\left(J_{\text {up }} \leqslant 3\right)$ CO lines are in the range of $10^{4}-10^{5}$ years (Cabrit \& Bertout 1992; Bontemps et al. 1996; Bachiller \& Tafalla 1999), which is considerably longer than the timescales associated with the protostellar energetics measured by both $L_{\mathrm{CO}}^{\mathrm{fir}}$ and $L_{\mathrm{bol}}$, and is a significant fraction of the protostellar lifetime. The measured $F_{\mathrm{CO}}$ and $L_{\mathrm{CO}}^{(\text {sub)mm }}$ are the average momentum and kinetic energy of the molecular flow over the observed dynamical age of the outflow ( $2 \%$ $-20 \%$ of the protostellar lifetime).

It is interesting to compare the functional dependence of $L_{\mathrm{CO}}^{\mathrm{fir}}$ with $L_{\mathrm{bol}}$ to that observed for $L_{\mathrm{CO}}^{(\mathrm{sub}) \mathrm{mm}}$. The mechanical luminosity of the outflow observed in low- $J$ CO lines, $L_{\mathrm{CO}}^{(\mathrm{sub}) \mathrm{mm}}$ is found to scale with $L_{\mathrm{bol}}$ as (Cabrit \& Bertout 1992)

$$
L_{\mathrm{CO}}^{\text {(sub)mm }} / L_{\mathrm{bol}}=4.3 \times 10^{-2} L_{\mathrm{bol}}{ }^{-0.2} .
$$

Both $L_{\mathrm{CO}}^{\mathrm{fir}}$ and $L_{\mathrm{CO}}^{(\text {sub)mm }}$ have a very similar dependence on $L_{\mathrm{bol}}$, over more than three orders of magnitude. However, $L_{\mathrm{CO}}^{\mathrm{fir}}$ is $\sim 70$ times lower than the $L_{\mathrm{CO}}^{(\text {sub)mm }}$ for a given $L_{\mathrm{bol}}$ (also see Lada 1985; Snell 1987; Wu et al. 2004). $L_{\mathrm{CO}}^{\text {fir }}$ and $L_{\mathrm{CO}}^{(\text {sub)mm }}$ are measured using independent techniques at different wavelengths, yet the remarkable similarity of their $L_{\mathrm{bol}}$ dependence and the large range over which the relation holds suggests that far-IR and (sub)millimeter $\mathrm{CO}$ emission are closely connected and probably caused by a common mechanism. The molecular outflows are driven by jets from protostars. As the jet propagates, it entrains ambient molecular material and accelerates it, shocking the molecular gas in the process. Part of the kinetic energy of the jet is dissipated in these shocks and part of it is transferred to the entrained molecular flow. The $L_{\mathrm{CO}}^{\mathrm{fir}}$ traces the currently shocked gas and is a measure of the energy dissipated by the jets in shocks. The $L_{\mathrm{CO}}^{(\text {sub }) \mathrm{mm}}$ is the average rate at which kinetic energy is injected into the molecular flow by the jet. Both $L_{\mathrm{CO}}^{\text {fir }}$ and $L_{\mathrm{CO}}^{(\text {sub)mm }}$ measure different manifestations of the same process and this possibly explains their similar $L_{\mathrm{bol}}$ dependence. The observed fact that $L_{\mathrm{CO}}^{\text {fir }}$ is 70 times lower than $L_{\mathrm{CO}}^{\text {(sub)mm }}$ would then mean that more of the kinetic energy in the jet goes into driving the molecular flow than is radiated away in shocks.

The measured $F_{\mathrm{CO}}$ and $L_{\mathrm{CO}}^{\text {(sub)mm }}$ are proportional to the mass ejection rate from the protostar smoothed over $10^{4}-10^{5}$ years, or, in other words, the time-averaged mass ejection rate from the protostar, $\left\langle\dot{M}_{\text {out }}\right.$. The average momentum flux of the 
molecular outflow $F_{\mathrm{CO}}$ is found to be correlated with protostellar evolutionary indicators. Bontemps et al. (1996) have found that $F_{\mathrm{CO}}$ is roughly proportional to the envelope mass, $M_{\text {env }}$, which they interpreted as a progressive decline of outflow activity (or equivalently mass ejection/accretion rate) during the protostellar accretion phase. Curtis et al. (2010) have confirmed the decline of $F_{\mathrm{CO}}$ with decreasing $M_{\mathrm{env}}$ and, in addition, have found that $F_{\mathrm{CO}}$ drops with increasing $T_{\mathrm{bol}}$ with a functional for $F_{\mathrm{CO}} \sim T_{\mathrm{bol}}-0.6$. These results indicate that both $\left\langle\dot{M}_{\text {out }}\right.$ and $\left\langle\dot{M}_{\text {acc }}\right\rangle$ steadily drop with protostellar age. Our results, however, show that $L_{\mathrm{CO}}^{\mathrm{fir}}$ is only weakly correlated with $T_{\text {bol }}$ and not correlated with $L_{\mathrm{bol}} / L_{\mathrm{smm}}$, indicating that the instantaneous mass-loss rate, $\dot{M}_{\text {out }}$, as measured by far-IR $L_{\mathrm{CO}}^{\text {fir }}$ does not show clear evidence of a steady decline with protostellar evolutionary tracers. This suggests that the instantaneous $\dot{M}_{\text {out }}$ and $\dot{M}_{\text {acc }}$ are not monotonically decreasing functions of system age. One possible explanation for this behavior is that the instantaneous accretion/ejection rate onto protostars is highly time variable and episodic, but the amplitude and/or frequency of this variability decreases with time such that the time-averaged accretion/ejection rates decline with protostellar age. Numerical simulations of the accretion history of protostars are consistent with such a time evolution for protostellar accretion rates (e.g., Vorobyov \& Basu 2008, 2010; Machida \& Hosokawa 2013).

We note, however, that the studies of the energetics derived from the low- $J \mathrm{CO}$ lines in the submillimeter and the highexcitation $\mathrm{CO}$ lines in the far-IR are carried out on different samples. These samples differ in the distribution of protostellar properties, and, in some cases, the observed SEDs from which the protostellar properties are estimated are not well sampled. For example, our sample has $L_{\mathrm{bol}}$, ranging from 0.07 to $275 L_{\odot}$, with a median value of $\sim 5 L_{\odot}$, whereas the sample of Bontemps et al. (1996) has a much narrower range in $L_{\mathrm{bol}}(0.2$ $-41 L_{\odot}$ ), with a slightly lower median of $2 L_{\odot}$. Although the Curtis et al. (2010) sample has an $L_{\text {bol }}$ range $\left(0.03-100 L_{\odot}\right)$ and median similar to our sample, the observed SEDs from which protostellar properties are computed are sparsely sampled, particularly the peak of the SED (see Hatchell et al. 2007b). Thus the correlations discussed above between various quantities will have to be demonstrated for the same sample, whose protostellar properties are well characterized, before one can draw robust conclusions about the time evolution of mass accretion/ejection in protostars.

\section{SUMMARY AND CONCLUSIONS}

We studied the evolution of far-IR high $-J\left(14 \leqslant J_{\text {up }} \leqslant 45\right)$ $\mathrm{CO}$ emission from protostars by analyzing the Herschel/PACS spectra of 50 embedded sources in the nearby star-forming regions observed by the HOPS and DIGIT Herschel key programs. We first constructed uniformly sampled SEDs for these sources. The peak of the SEDs are well sampled with Herschel/PACS photometric observations at 70, 100, and $160 \mu \mathrm{m}$. We then computed the bolometric luminosity, $L_{\mathrm{bol}}$, bolometric temperature, $T_{\mathrm{bol}}$, and fractional submillimeter luminosity, $L_{\mathrm{bol}} / L_{\mathrm{smm}}$ of the protostars in our sample in a uniform way from the observed SEDs. The protostars in our sample have a large range in $L_{\mathrm{bol}}$ (more than three orders of magnitude) and are at various stages of evolution. For this sample, we searched for correlations between far-IR CO line luminosities and various protostellar properties. Our main results and conclusions are summarized below.
1. We find a strong and tight correlation between far-IR CO luminosity, $L_{\mathrm{CO}}^{\mathrm{fir}}$, and the bolometric luminosity, $L_{\mathrm{bol}}$, of the protostars. The far-IR CO luminosity, $L_{\mathrm{CO}}^{\text {fir }}$ scales with $L_{\text {bol }}$ as $L_{\mathrm{CO}}^{\mathrm{fir}} \propto L_{\mathrm{bol}}^{0.7}$. This correlation extends over more than three orders of magnitude in both quantities, with a mean dispersion from the relation of less than a factor of two.

2. We find a weak correlation between $L_{\mathrm{CO}}^{\mathrm{fir}}$ and $T_{\text {bol }}$, but no correlation is found between $L_{\mathrm{CO}}^{\mathrm{fir}}$ and $L_{\mathrm{bol}} / L_{\mathrm{smm}}$.

3. FIR CO emission from protostars trace the currently shocked gas by jets/outflows, the cooling and dynamical timescales for which are $\leqslant 200$ years, significantly shorter than the protostellar lifetimes. $L_{\mathrm{CO}}^{\mathrm{fir}}$, is proportional to the instantaneous mechanical luminosity of the jet/outflow, which scales with the instantaneous mass-loss rate, $\dot{M}_{\text {out }}$. The correlation between $L_{\mathrm{CO}}^{\mathrm{fir}}$ and $L_{\mathrm{bol}}$, then, is indicative of instantaneous $\dot{M}_{\text {out }}$ tracking instantaneous $\dot{M}_{\text {acc }}$.

4. The lack of (or weak) correlation between $L_{\mathrm{CO}}^{\text {fir }}$ and evolutionary indicators $T_{\mathrm{bol}}$ and $L_{\mathrm{bol}} / L_{\mathrm{smm}}$ suggests that $\dot{M}_{\text {out }}$ and, therefore, $\dot{M}_{\text {acc }}$ do not show any clear evolutionary trend. Thus our results are consistent with mass accretion/ejection in protostars being episodic.

5. We compared our results with those found for the mechanical luminosity $\left(L_{\text {mech }}\right)$ and the momentum flux or outflow force $\left(F_{\mathrm{CO}}\right)$ of the molecular outflows observed in low- $J\left(J_{\text {up }} \leqslant 3\right)$ CO lines at (sub-)millimeter wavelengths. The functional dependence of $L_{\mathrm{CO}}^{\mathrm{fir}}$ on $L_{\mathrm{bol}}$ that we find is similar to that found for the mechanical luminosity, $L_{\mathrm{CO}}^{(\mathrm{sub}) \mathrm{mm}}$, of molecular outflows observed in low-excitation CO lines. The observed similarity and the large range over which the relations hold suggest that far-IR and (sub)millimeter $\mathrm{CO}$ emission are closely connected.

Studies of molecular outflows in low- $J\left(J_{\text {up }} \leqslant 3\right)$ also indicate that the time-averaged mass ejection/accretion rate steadily declines during the protostellar phase (Bontemps et al. 1996). Our results, on the other hand, suggest that the instantaneous accretion/ejection rate does not show a clear evolutionary trend. One possible explanation for this is that mass-accretion/ejection rate in protostars is highly time variable and episodic, but the amplitude and/or frequency of this variability decreases with time such that the time averaged accretion/ejection rate declines with system age.

These correlations will have to be demonstrated for the same sample from a homogeneous set of observations before the detailed behavior of the time evolution of mass accretion/ejection in protostars can be confirmed.

Support for this work, part of the Herschel Open Time Key Project Program, was provided by NASA through an award issued by the Jet Propulsion Laboratory, California Institute of Technology. This work was supported by NSF grant AST1109116 to the University of Texas at Austin. This work is based on observations made with the Herschel Space Observatory, a European Space Agency Cornerstone Mission with significant participation by NASA; it is also on observations made with the Spitzer Space Telescope, which is operated by the Jet Propulsion Laboratory, California Institute of Technology (Caltech), under a contract with NASA. We also include data from the Atacama Pathfinder Experiment, a collaboration between the Max-Planck Institut für Radio-astronomie, the European Southern Observatory, and 
the Onsala Space Observatory. This publication makes use of data products from the Two Micron All Sky Survey, which is a joint project of the University of Massachusetts and the Infrared Processing and Analysis Center/Caltech, funded by NASA and the NSF.

\section{REFERENCES}

Adams, J. D., Herter, T. L., Osorio, M., et al. 2012, ApJL, 749, L24

Akritas, M. G., Murphy, S. A., \& Lavalley, M. P. 1995, J. Am. Stat. Assoc., 90, 170

André, P., Men'shchikov, A., Bontemps, S., et al. 2010, A\&A, 518, L102

André, P., Ward-Thompson, D., \& Barsony, M. 1993, ApJ, 406, 122

André, P., Ward-Thompson, D., \& Barsony, M. 2000, in Protostars and Planets IV, ed. V. Mannings, A. P. Boss, \& S. S. Russell (Tucson, AZ: Univ. Arizona Press), 59

Arce, H. G., Shepherd, D., Gueth, F., et al. 2007, in Protostars and Planets V, ed. V. B. Reipurth, D. Jewitt, \& K. Keil (Tucson, AZ: Univ. Arizona Press), 245

Audard, M., Ábrahám, P., Dunham, M. M., et al. 2014, in Protostars and Planets VI, ed. H. Beuther et al. (Tucson, AZ: Univ. Arizona Press), 387

Bachiller, R., \& Tafalla, M. 1999, in the Origin of Stars and Planetary Systems, ed. C. J. Lada \& N. D. Kylafis (Dordrecht: Kluwer), 227

Bally, J., \& Lada, C. J. 1983, ApJ, 265, 824

Bally, J., Reipurth, B., \& Davis, C. J. 2007, in Protostars and Planets V, ed. B. Reipurth, D. Jewitt, \& K. Keil (Tucson, AZ: Univ. Arizona Press), 215 Bontemps, S., André, P., Terebey, S., \& Cabrit, S. 1996, A\&A, 311, 858

Brown, B. W., Hollander, M., \& Korwar, R. M. 1974, in Reliability and Biometry, ed. F. Proschan \& R. J. Serfling (Philadelphia: SIAM), 327

Cabrit, S., \& Bertout, C. 1992, A\&A, 261, 274

Calvet, N., \& Gullbring, E. 1998, ApJ, 509, 802

Calvet, N., Muzerolle, J., Briceño, C., et al. 2004, AJ, 128, 1294

Chen, H., Myers, P. C., Ladd, E. F., \& Wood, D. O. S. 1995, ApJ, 445, 377

Cieza, L. A., Olofsson, J., Harvey, P. M., et al. 2013, ApJ, 762, 100

Curtis, E. I., Richer, J. S., Swift, J. J., \& Williams, J. P. 2010, MNRAS, 408, 1516

Dionatos, O., Jørgensen, J. K., Green, J. D., et al. 2013, A\&A, 558, A88

Dunham, M. 2010, OT1_mdunham_1: Understanding the Protostellar Mass Accretion Process: Herschel 100-500 micron Photometry of Low Luminosity Embedded Protostars, Herschel Space Observatory Proposal, id. 1359

Dunham, M. M., Arce, H. G., Mardones, D., et al. 2014a, ApJ, 783, 29

Dunham, M. M., Evans, N. J., II, Terebey, S., Dullemond, C. P., \& Young, C. H. 2010, ApJ, 710, 470

Dunham, M. M., Stutz, A. M., Allen, L. E., et al. 2014, in Protostars and Planets VI, ed. H. Beuther et al. (Tucson, AZ: Univ. Arizona Press), 195

Dunham, M. M., \& Vorobyov, E. I. 2012, ApJ, 747, 52

Evans, N. J., Dunham, M. M., Jørgensen, J. K., et al. 2009, ApJS, 181, 321

Fedele, D., Bruderer, S., van Dishoeck, E. F., et al. 2013, A\&A, 559, A77

Feigelson, E., \& Babu, G. 2012, Modern Statistical Methods for Astronomy: With R Applications (Cambridge: Cambridge Univ. Press)

Fischer, W. J., Megeath, S. T., Ali, B., et al. 2010, A\&A, 518, L122

Fischer, W. J., Megeath, S. T., Stutz, A. M., et al. 2013, AN, 334, 53

Fischer, W. J., Megeath, S. T., Tobin, J. J., et al. 2012, ApJ, 756, 99

Frank, A., Ray, T. P., Cabrit, S., et al. 2014, in Protostars and Planets VI, ed. H. Beuther et al. (Tucson, AZ: Univ. Arizona Press), 451

Furlan, E., Fischer, W. J., Ali, B., et al. 2016, ApJS, 224, 5

Furlan, E., Megeath, S. T., Osorio, M., et al. 2014, ApJ, 786, 26

Gonzalez-Garcia, B., Manoj, P., Watson, D. M., et al. 2016, A\&A, submitted (arXiv:1608.04964)

Green, J. D., Evans, N. J., II, Kóspál, Á., et al. 2013a, ApJ, 772, 117

Green, J. D., Evans, N. J., II, Jørgensen, J. K., et al. 2013b, ApJ, 770, 123

Green, J. D., Hartmann, L., Calvet, N., et al. 2006, ApJ, 648, 1099

Green, J. D., Yang, Y.-L., Evans, N. J., II, et al. 2016, AJ, 151, 75

Gullbring, E., Hartmann, L., Briceno, C., \& Calvet, N. 1998, ApJ, 492, 323

Hartmann, L., Calvet, N., Gullbring, E., \& D'Alessio, P. 1998, ApJ, 495, 385

Hartmann, L. 2009, Accretion Processes in Star Formation (2nd edn; Cambridge: Cambridge Univ. Press)

Hartmann, L., \& Kenyon, S. J. 1996, ARA\&A, 34, 207

Hatchell, J., Fuller, G. A., \& Richer, J. S. 2007a, A\&A, 472, 187
Hatchell, J., Fuller, G. A., Richer, J. S., Harries, T. J., \& Ladd, E. F. 2007b, A\&A, 468, 1009

Herbig, G. H. 1977, ApJ, 217, 693

Herczeg, G. J., \& Hillenbrand, L. A. 2008, ApJ, 681, 594

Hollenbach, D. J., Chernoff, D. F., \& McKee, C. F. 1989, in ESA Special Publication 290, Infrared Spectroscopy in Astronomy, ed. E. Böhm-Vitense, 245

Karska, A., Herczeg, G. J., van Dishoeck, E. F., et al. 2013, A\&A, 552, A141 Kenyon, S. J. 1995, RMxAA, 27, 237

Kenyon, S. J., Hartmann, L. W., Strom, K. M., \& Strom, S. E. 1990, AJ, 99, 869

Kim, M. K., Hirota, T., Honma, M., et al. 2008, PASJ, 60, 991

Krause, O. 2010, in From Stars to Galaxies: Connecting our Understanding of Star and Galaxy Formation, 21

Lada, C. J. 1985, ARA\&A, 23, 267

Launhardt, R., Stutz, A. M., Schmiedeke, A., et al. 2013, A\&A, 551, A98

Lee, J.-E., Lee, J., Lee, S., Evans, N. J., II, \& Green, J. D. 2014a, ApJS, 214, 21

Lee, L. 2013, NADA: Nondetects And Data Analysis for environmental data, R package version 1.5-6, https://CRAN.R-project.org/package=NADA

Lee, S., Lee, J.-E., Bergin, E. A., \& Park, Y.-S. 2014b, ApJS, 213, 33

Machida, M. N., \& Hosokawa, T. 2013, MNRAS, 431, 1719

Manoj, P., Watson, D. M., Neufeld, D. A., et al. 2013, ApJ, 763, 83

Matt, S., \& Pudritz, R. E. 2005, ApJL, 632, L135

Matt, S., \& Pudritz, R. E. 2008, ApJ, 681, 391

Matuszak, M., Karska, A., Kristensen, L. E., et al. 2015, A\&A, 578, 20

Meeus, G., Salyk, C., Bruderer, S., et al. 2013, A\&A, 559, A84

Menten, K. M., Reid, M. J., Forbrich, J., \& Brunthaler, A. 2007, A\&A, 474,515

Muzerolle, J., Calvet, N., \& Hartmann, L. 1998a, ApJ, 492, 743

Muzerolle, J., Calvet, N., \& Hartmann, L. 2001, ApJ, 550, 944

Muzerolle, J., D’Alessio, P., Calvet, N., \& Hartmann, L. 2004, ApJ, 617, 406

Muzerolle, J., Hartmann, L., \& Calvet, N. 1998b, AJ, 116, 2965

Myers, P. C., \& Ladd, E. F. 1993, ApJL, 413, L47

Najita, J. R., \& Shu, F. H. 1994, ApJ, 429, 808

Nisini, B., Santangelo, G., Giannini, T., et al. 2015, ApJ, 801, 121

Pelletier, G., \& Pudritz, R. E. 1992, ApJ, 394, 117

Plunkett, A. L., Arce, H. G., Corder, S. A., et al. 2015, ApJ, 803, 22

R Core Team 2015, R: A Language and Environment for Statistical Computing, R Foundation for Statistical Computing, Vienna, Austria

Reipurth, B., \& Aspin, C. 2010, in Evolution of Cosmic Objects through their Physical Activity, ed. H. A. Harutyunian, A. M. Mickaelian, \& Y. Terzian (Yerevan: Gitutyun), 19

Richer, J. S., Shepherd, D. S., Cabrit, S., Bachiller, R., \& Churchwell, E. 2000, in Protostars and Planets IV, ed. V. Mannings, A. P. Boss, \& S. S. Russell (Tucson, AZ: Univ. Arizona Press), 867

Rodriguez, L. F., Carral, P., Ho, P. T. P., \& Moran, J. M. 1982, ApJ, 260, 635

Safron, E. J., Fischer, W. J., Megeath, S. T., et al. 2015, ApJL, 800, L5

Sandstrom, K. M., Peek, J. E. G., Bower, G. C., Bolatto, A. D., \& Plambeck, R. L. 2007, ApJ, 667, 1161

Sen, P. K. 1968, J. Am. Stat. Assoc., 63, 1379

Shu, F., Najita, J., Ostriker, E., et al. 1994, ApJ, 429, 781

Snell, R. L. 1987, in IAU Symp. 115, Star Forming Regions, ed. M. Peimbert \& J. Jugaku (Dordrecht: Reidel), 213

Stanke, T., Stutz, A. M., Tobin, J. J., et al. 2010, A\&A, 518, L94

Sturm, B., Bouwman, J., Henning, T., et al. 2013, A\&A, 553, A5

Stutz, A., Launhardt, R., Linz, H., et al. 2010, A\&A, 518, L87

Stutz, A. M., \& Kainulainen, J. 2015, A\&A, 577, L6

Stutz, A. M., Tobin, J. J., Stanke, T., et al. 2013, ApJ, 767, 36

Takahashi, S., Saito, M., Ohashi, N., et al. 2008, ApJ, 688, 344

Tobin, J. 2010, OT1_jtobin_1: Protostellar Envelopes Resolved Inside and Out: A Close Look in the Far-IR, Herschel Space Observatory Proposal, id. 752

Tobin, J. J., Hartmann, L., Chiang, H.-F., et al. 2012, Natur, 492, 83

Tobin, J. J., Stutz, A. M., Manoj, P., et al. 2016, arXiv:1607.00787

Tobin, J. J., Stutz, A. M., Megeath, S. T., et al. 2015, ApJ, 798, 128

van Kempen, T. A., Green, J. D., Evans, N. J., et al. 2010, A\&A, 518, L128

Vorobyov, E. I., \& Basu, S. 2008, ApJL, 676, L139

Vorobyov, E. I., \& Basu, S. 2010, ApJ, 719, 1896

Wardle, M., \& Koenigl, A. 1993, ApJ, 410, 218

Watson, D. M. 1985, PhST, 11, 33

Wu, Y., Wei, Y., Zhao, M., et al. 2004, A\&A, 426, 503 